\title{
Irreducibility of analytic arc-sections of hypersurface singularities.
}

\author{
Miguel Angel Marco-Buzunariz ${ }^{* 1}$ and Maria Pe Pereira ${ }^{\dagger 2}$ \\ 1 Departamento de Matemáticas, Universidad de Zaragoza, \\ C/ Pedro Cerbuna 12, 50003, Zaragoza, , \\ mmarco@unizar.es \\ 2 Departamento de Álgebra, Geometría y Topología and Instituto de Matemática \\ Interdisciplinar, Facultad de Ciencias Matemáticas- UCM, \\ Plaza de Ciencias, 3, Ciudad Universitaria, 28040 Madrid, \\ maria.pe@mat.ucm.es
}

February 10, 2020

\begin{abstract}
We explore the existence of irreducible and reducible arc-sections in an irreducible hypersurface singularity germ along finite projections.

In particular we provide examples of irreducible isolated hypersurface singularities for which no irreducible arc-sections exist, and show that reducible ones always exist. Moreover, we give an algorithm to check if a given projection allows irreducible arc-sections, and find them if they exist.
\end{abstract}

\section{Introduction}

We work on the following basic question:

Question. If all the intersections of a hypersurface singularity germ $(X, 0) \subset$ $\left(\mathbb{C}^{n+1}, 0\right)$ by a surface are reducible, can we conclude that $(X, 0)$ is reducible?

In the same direction, it is clear that, for a given hypersuface germ $(X, 0)$, the existence of an irreducible section with a surface that is not tangent to $X$ and that is not contained in the singular locus of $(X, 0)$ is a criterium for the irreducibility of $(X, 0)$.

From a practical point of view it is natural trying to reduce the study to sections with surfaces that are the preimage by a single linear projection $\pi$ : $\mathbb{C}^{n} \rightarrow \mathbb{C}^{n-1}$ of an arc in $\mathbb{C}^{n-1}$. More precisely, given a hypersurface germ singularity $(X, \overline{0}) \subseteq\left(\mathbb{C}^{n+1}, \overline{0}\right)$ defined by the equation $F\left(x_{1}, \ldots, x_{n+1}\right)=0$, and

\footnotetext{
*Partially supported by MTM2016-76868-C2-2-P and Grupo "Investigación en Educación Matemática" of Gobierno de Aragón/Fondo Social Europeo.

†Partially supported by MTM2017-89420-P, MTM2016-76868-C2-1-P and ERC Consolidator Grant NMST.
} 
an injective arc $\gamma:(\mathbb{C}, 0) \rightarrow\left(\mathbb{C}^{n}, \overline{0}\right)$, we say that the plane curve defined by $F\left(\gamma(t), x_{n+1}\right)=0$ is an arc-section of $(X, \overline{0})$.

In this article, we begin to study, mainly in the surface case, the existence of irreducible and reducible arc sections, how to detect them and how sensible the problem is after a change of coordinates.

Some examples, may lead to wrong conclusions. For example, consider a suspension singularity given by an equation of the form $z^{m}=f(x, y)$ with $f(x, y)$ an irreducible germ of multiplicity $d$ with g.c.d. $(m, d)=1$.

Given any generic line $L$ in the $(x, y)$-plane parametrized as $(a t, b t)$, we consider the plane curve germ given by $z^{m}=f(a t, b t)$. It is easy to see that this plane branch is irreducible since the associated Newton polygon consists on a unique bounded segment with no integer points in its interior.

We wonder if this is the general case. That is:

Question. Let $(X, \overline{0})$ be an irreducible hypersurface germ defined in $\mathbb{C}^{n+1}$ by the equation $F\left(x_{1}, \ldots, x_{n+1}\right)=0$. Do there exist a linear projection $\pi: \mathbb{C}^{n+1} \rightarrow$ $\mathbb{C}^{n}$, say $\left(x_{1}, \ldots, x_{n+1}\right) \mapsto\left(x_{1}, . ., x_{n}\right)$ with $\left.\pi\right|_{(X, \overline{0})}$ finite and an injective arc $\gamma:(\mathbb{C}, 0) \rightarrow\left(\mathbb{C}^{n}, \overline{0}\right)$ such that $F\left(\gamma(t), x_{n+1}\right)=0$ is an irreducible plane curve germ?

We give a negative answer to this question in Proposition 4.2, by showing a family of counterexamples. This also answers negatively the initial question. In Section 5 we prove that reducible arc-sections always exist for every finite projection $\left.\pi\right|_{X, \overline{0}}$, and moreover, for every finite projection $\left.\pi\right|_{X, \overline{0}}$, there are arcsections with as many components as the degree of $\left.\pi\right|_{X, \overline{0}}$.

We also see that, apart from the cases where the projectivization of the tangent cone of $(X, \overline{0})$ is a union of lines, the generic arc is never irreducible.

In Section 7 we show an algorithm to determine whether irreducible arcsections over an arc not contained in the discriminant exists or not; and to find them in case they do (see Section 7 and useful information for a shortcut in Section 6). In the final section we see in an example how the behavior over the discriminant can change by moving the projection.

The second author thanks A. Płoski and E. García Barroso for asking about the existence of irreducible arc sections for a fixed projection, which motivated this work.

\section{Notation and general setting}

Let $(X, \overline{0})$ be a hypersurface germ defined by $F\left(x_{1}, \ldots, x_{n+1}\right)=0$. Let $m$ be the multiplicity of $(X, \overline{0})$. Let $\pi: \mathbb{C}^{n+1} \rightarrow \mathbb{C}^{n}$ be a linear projection such that $\left.\pi\right|_{(X, \overline{0})}$ is finite (see Remark 3.4). After a linear change of coordinates we can assume that $\pi$ is given by

$$
\left(x_{1}, \ldots, x_{n+1}\right) \mapsto\left(x_{1}, . ., x_{n}\right) .
$$

We say that the projection is transverse (with respect to $(X, \overline{0})$ ) if it is in a direction not contained in the tangent cone of $(X, \overline{0})$. Otherwise we say that it is non-transverse.

Let $\Delta$ denote the discriminant of $\left.\pi\right|_{X}$. Note that the restriction $\left.\pi\right|_{X \backslash \pi^{-1}(\Delta)}$ is a regular covering of certain degree $d$. Then, given a loop $\delta$ in $\mathbb{C}^{n} \backslash \Delta$ we have 
the monodromy of the covering along $\delta$ which gives an element of $\operatorname{Sym}(d)$, the permutation group of $d$ points.

Since $\left.\pi\right|_{X, \overline{0}}$ is finite, we can assume $F\left(x_{1}, \ldots, x_{n+1}\right)$ is a Weierstrass polynomial in $x_{n+1}$ of degree $d$. Moreover, if the projection is transverse then $d=m$. In the other case, we have $d>m$.

We denote by $C_{X, \overline{0}}$ the tangent cone of $(X, \overline{0})$ seen, either as a conic subvariety germ of $\left(\mathbb{C}^{n+1}, \overline{0}\right)$ or a subvariety of $\mathbb{C P}^{n}$.

Given $\pi: \mathbb{C}^{n+1} \rightarrow \mathbb{C}^{n}$ as above, we denote by $p_{\pi}$ the point in $\mathbb{C P}^{n}$ that gives the projection direction. We denote by $\bar{\pi}: \mathbb{C P}^{n} \backslash\left\{p_{\pi}\right\} \rightarrow \mathbb{C P}^{n-1}$ the projection from $p_{\pi}$.

Given a subspace $V$ of $\mathbb{C P}^{n}$, we denote by $\langle V\rangle$ the linear subspace of $\mathbb{C}^{n+1}$ that it generates.

\section{Arc-sections.}

In this section we fix notation and definitions regarding arcs and arc-sections.

Definition 3.1. An arc in $\left(\mathbb{C}^{n}, \overline{0}\right)$ is a holomorphic mapping germ $\gamma:(\mathbb{C}, 0) \rightarrow$ $\left(\mathbb{C}^{n}, \overline{0}\right)$. A representative of an arc $\gamma$ is the restriction of $\gamma$ to some closed disk $\mathbb{D} \subset \mathbb{C}$ centered at $0 \in \mathbb{C}$ contained in the domain of $\gamma . A$ smooth arc is an arc $\gamma$ such that $\gamma^{\prime}(0) \neq \overline{0}$. We say that an arc $\gamma(t)$ is transversal to a subvariety $Y$ of $\left(\mathbb{C}^{n}, \overline{0}\right)$ at $t_{0} \in \mathbb{C}$ if $\gamma^{\prime}\left(t_{0}\right) \neq \overline{0}$ and $\gamma^{\prime}\left(t_{0}\right)$ is not contained in the tangent space of $Y$ at $\gamma\left(t_{0}\right)$. We say that $\gamma(t)$ is transversal to $Y$ if it is transversal at any point.

In general we will only consider injective representatives $\left.\gamma\right|_{\mathbb{D}}$ (which always exist for generically 1:1 arcs). In particular we will assume that $\mathbb{D}$ is small enough so that $\left(\left.\gamma\right|_{\mathbb{D}}\right)^{-1}(\overline{0})=\{0\}$. Then, note that $\left.\gamma\right|_{\partial \mathbb{D}}$ is a simple loop in $\mathbb{C}^{n} \backslash\{\overline{0}\}$.

Lemma 3.2. Let $(X, \overline{0}), F\left(x_{1}, \ldots, x_{n+1}\right) \in \mathbb{C}\left\{x_{1}, \ldots, x_{n+1}\right\}$ and $\pi$ be as above. Given an arc $\alpha:(\mathbb{C}, 0) \rightarrow\left(\mathbb{C}^{n}, \overline{0}\right)$ whose image is not completely contained in the discriminant $\Delta$, the following are equivalent:

1. $F\left(\alpha(t), x_{n+1}\right)=0$ defines an irreducible germ at $(0,0) \in \mathbb{D} \times \mathbb{C}$

2. $\pi^{-1}(\alpha(\mathbb{D})) \cap X$ is an irreducible germ at $\overline{0} \in \mathbb{C}^{n+1}$ for an injective representative $\alpha: \mathbb{D} \rightarrow \mathbb{C}^{n}$ such that $\alpha(\mathbb{D}) \cap \Delta=\{\overline{0}\}$

3. the monodromy along $\alpha(\partial \mathbb{D})$ is transitive (with $\left.\alpha\right|_{\mathbb{D}}$ a representative as in (2)), that is, it gives a d-cycle of $\operatorname{Sym}(d)$.

Proof. To see $(1) \Leftrightarrow(2)$ we observe that the mapping

$$
\begin{aligned}
\mathbb{C}^{2} & \rightarrow \mathbb{C}^{n+1} \\
\left(t, x_{n+1}\right) & \mapsto\left(\alpha(t), x_{n+1}\right)
\end{aligned}
$$

is injective restricted to $\mathbb{D} \times \mathbb{C}$ since $\left.\alpha\right|_{\mathbb{D}}$ is injective. Then, its restriction to the germ $F\left(\alpha(t), x_{n+1}\right)=0$ is a homeomorphism onto $\pi^{-1}(\alpha(\mathbb{D})) \cap X$. Then, one of them is irreducible if and only if so is the other.

To see $(2) \Leftrightarrow(3)$ we note that the singular locus of $\pi^{-1}(\alpha(\mathbb{D})) \cap X$ is just the origin. Then, $\pi^{-1}(\alpha(\mathbb{D})) \cap X$ is irreducible if and only if it remains connected 
after removing the origin. The map $\left.\pi\right|_{\pi^{-1}(\alpha(\mathbb{D} \backslash\{0\})) \cap X}$ onto $\alpha(\mathbb{D} \backslash\{0\})$ is a cover over a space that can be retracted to the simple closed loop $\alpha(\partial \mathbb{D})$. So $\pi^{-1}(\alpha(\mathbb{D} \backslash\{0\})) \cap X$ is connected if and only if the monodromy along $\alpha(\partial \mathbb{D})$ is transitive.

Note that equivalence of (1) and (2) is also true if the arc is contained in the discriminant.

Last lemma justifies the following definition and allows us to think geometrically about the problem.

Definition 3.3. Let $\pi: \mathbb{C}^{n+1} \rightarrow \mathbb{C}^{n}$ be a linear projection such that $\left.\pi\right|_{(X, \overline{0})}$ is finite. An arc-section of $(X, \overline{0})$ is a section of $(X, \overline{0})$ of the form $\pi^{-1}(\alpha(\mathbb{D})) \cap X$ for certain injective arc representative $\alpha: \mathbb{D} \rightarrow \mathbb{C}^{n}$ that is either contained in the discriminant $\Delta$ of $\left.\pi\right|_{X}$ or $\alpha(\mathbb{D}) \cap \Delta=\{\overline{0}\}$.

So, our main question is about the existence or non-existence of irreducible and reducible arc-sections for irreducible hypersurface germs.

Note that if $\alpha(\mathbb{D})$ is contained in $\Delta$, then the arc-section is non-reduced (that is, there are embedded components). So, for arcs in $\mathbb{C}^{n}$ whose image is contained in the discriminant, we ask whether they give irreducible arc-sections considered with its reduced structure.

Remark 3.4. As we said, we always assume $\left.\pi\right|_{X, \overline{0}}$ is finite/proper, which is equivalent to $\pi^{-1}(\overline{0})=\{\overline{0}\}$. If the projection is not proper, then an arc-section either has $\pi^{-1}(\overline{0})$ as irreducible component or is the whole surface $\pi^{-1}(\alpha(\mathbb{D}))$. Note that a non-trasverse linear projection, that is a projection in a direction contained in the tangent cone of $(X, \overline{0})$, is always proper in case this line of the tangent cone is not contained in $X$.

\section{Looking at the tangent cone of an arc-section.}

Here we will see some necessary conditions on the tangent cone of $(X, \overline{0})$ for the existence of irreducible arc-sections.

Lemma 4.1. Let $\pi: \mathbb{C}^{n+1} \rightarrow \mathbb{C}^{n}$ be a linear projection such that $\left.\pi\right|_{(X, \overline{0})}$ is finite. Let $L$ be a line in $\mathbb{C P}^{n}$ containing the projection direction. Assume the intersection (with reduced structure) of $L$ and $C_{X, \overline{0}}$ in $\mathbb{C P}^{n}$ is a union of isolated points. Consider any arc in $\left(\mathbb{C}^{n}, \overline{0}\right)$ with tangent at the origin in the direction given by a point of $L$. Then the reduced tangent cone of the corresponding arcsection is given by the intersection $C_{X, \overline{0}} \cap L$. In particular, if the intersection is 2 or more different points then the arc section is reducible.

Proof. Note first that the tangent space of a set of the form $\pi^{-1}(\alpha(\mathbb{D}))$ is the plane generated by the projection direction of $\pi$ and the tangent of the arc $\alpha$. Viewed in $\mathbb{C P}^{n}$, it is the line joining the corresponding points.

After the blowing up of the origin of $\mathbb{C}^{n+1}$, we get the strict transform of the set $\pi^{-1}(\alpha(\mathbb{D}))$ and the hypersurface $(X, \overline{0})$. Their common intersection with the exceptional divisor is a set of isolated points by hypothesis. Around any of these points we have the intersection of a hypersurface and a surface that is not contained in it (because the projection is proper) in a smooth space. Then, the intersection is a (not necessarily irreducible) curve. Hence, for any point of $C_{X, \overline{0}} \cap L$ we have at least one component of the arc section. 
Note that the only case in which the hypothesis of the previous proposition are not satisfied is when $L$ is contained in $C_{X, \overline{0}}$.

Note also that, if $C_{X, \overline{0}}$ intersects every line in more that one point, no irreducible arc-sections can exist. This happens, for instance, if $C_{X, \overline{0}}$ is a smooth curve with degree bigger than three and no hyperflexes (that is, no points where the tangent line has contact order bigger than three).

Then, we can summarize our conclusion in two propositions:

Proposition 4.2. There exist irreducible hypersurface germs $(X, \overline{0})$ with all its arc-sections being reducible. Moreover, if we apply any analytic change of coordinates, the resulting germs also satisfy this property.

Corollary 4.3. There exist irreducible hypersurface germs $(X, \overline{0})$ such that the intersection with any smooth surface germ $(Y, \overline{0})$ is reducible.

Example 4.4. The smooth quartic given by $x^{4}+y^{4}+x^{2} z^{2}+y z^{3}+z^{4}=0$ does not have hyper-flexes, and then it is an explicit example. Note that the set of smooth quartics without hyperflexes is Zariski open in the set of quartics. In this example, the equation considered in affine coordinates gives the germ in $\left(\mathbb{C}^{3}, \overline{0}\right)$, and the same equation considered as homogenous coordinates in $\mathbb{C P}^{2}$ gives its tangent cone.

Example 4.5. Another example is any surface with smooth irreducible tangent cone of degree greater or equal than 4 and no hyperflexes. For every degree this is a Zariski open set of the set of surfaces of that degree. Other examples are given by surfaces whose tangent cone is the union of two non-tangent conics.

Proposition 4.6. A necessary condition for the existence of an irreducible arcsection in $(X, \overline{0})$ is that there exists a line $L$, which may be contained in one irreducible component of $C_{X, \overline{0}}$, that meets any component of $C_{X, \overline{0}}$ that does not contain it (if any) in exactly one point. This point would be an intersection point of all the irreducible components of $C_{X, \overline{0}}$, in case there are more than one.

When this necessary condition is achieved, that is, in case there exists such a line $L$, then the starting point to look for irreducible arcs sections is the following:

(i) consider a projection $\pi$ in the direction of a point $p_{\pi} \in L$. Since we ask the projection to be proper, we have to require the line $\left\langle p_{\pi}>\subset \mathbb{C}^{n+1}\right.$ to not be contanined in $(X, \overline{0})$;

(ii) once the projection is fixed, the only arcs that may lead to irreducible arc sections must have tangent cone equal to $\bar{\pi}\left(L^{\prime}\right)$ with $L^{\prime}$ some line as in Corollary 4.6 passing through the projection point. In Section 7.1, we give an alternative description of the possible tangent cones in some cases.

Remark 4.7. We can easily summarize, in the surface case, the cases in which such a line $L$ can exist. There are 5 cases for $C_{X, \overline{0}}$ with reduced structure, that are (a) a line, (b) a union of lines meeting in a common point p, (c) a conic, (d) an irreducible surface of degree $d>2$ that intersects some tangent line with multiplicity $d$ and (e) a reducible surface with a common point $p$ of all 
its components (and such that there is a line through $p$ that is a tangent line of all the components of degree $d \geq 2$ of maximal intersection multiplicity).

We can saee that (d) is never the case if $C_{X, \overline{0}}$ is a generic variety of degree greater or equal than 4 .

We retake the examples in the introduction, that cover the cases (a)-(b) in the previous remark:

Example 4.8. For the surface $z^{d}=f(x, y)$ with $\operatorname{ord}(f)=m>d$ with $g c d(d, m)=$ 1. The tangent cone is $z=0$. Then, we can expect irreducible arc sections for any projection transverse to $z=0$ and in any direction. In particular for the projection in the direction of $z$ one can check easily that (at,bt) gives irreducible arc section whenever the initial form of $f$ evaluated at $(a, b)$ doesn't vanish.

Example 4.9. In the case of $z^{d}=f(x, y)$ with ord $(f)=m<d$ and $g c d(d, m)=$ 1 , we have that the tangent cone can be in general given by a union of lines (in $\left(\mathbb{P}^{2}\right)$. So, we can expect irreducible arc sections in any direction for a nontransverse projection. Then, for example, for the projection to the XY-plane, for generic $(a, b)$, it is easy to see that $(a t, b t)$ give irreducible arc sections.

Looking at the possibilities (a)-(e) for the case of surfaces in Remark 4.7. we see that the existence of irreducible arc-sections for the generic projection may only hold in the cases (a)-(c) and cases in (e) where the tangent cone is a product of tangent cones as in $(a)-(c)$ (as for example $C_{X, \overline{0}}$ being a union of a conic and some lines through a given point). A natural question would be wether the existance of an irreducible arc-section in these cases is invariant by changing the generic projection or even by analytical change of coordinates.

\section{Existence of reducible arc-sections with $d$ com- ponents}

Let $(X, \overline{0})$ be a surface germ in $\left(\mathbb{C}^{3}, \overline{0}\right)$ and let $\pi: \mathbb{C}^{3} \rightarrow \mathbb{C}^{2}$ be a projection so that $\left.\pi\right|_{X}$ is finite.

Definition 5.1. We define transversal monodromies as follows:

(a) we say that the monodromy of a loop $\delta$ parametrizing the boundary of a small disk transversal to a component of $\Delta$ is the transversal monodromy associated to the component of $\Delta$ it meets.

(b) Given any exceptional component $E_{i}$ of a composition of blow ups over the origin of $\left(\mathbb{C}^{2}, 0\right)$, we can consider any arc $\gamma$ in $\mathbb{C}^{2}$ with transversal lifting through $E_{i}$ not meeting any other component of the total transform of $\Delta$. Given $\mathbb{D}$ such that $\gamma(\mathbb{D}) \cap \Delta=\{\overline{0}\}$, the monodromy of $\left.\gamma\right|_{\partial \mathbb{D}}$ is called the transverse monodromy associated to the divisor $E_{i}$.

Take an embedded resolution of $\left(\mathbb{C}^{2}, \Delta\right)$. Let $\Delta^{*}$ be the total transform of $\Delta$.

Take a normal crossing point in $\Delta^{*}$. It has two local branches, $F_{1}$ and $F_{2}$. Let $\sigma_{1}$ and $\sigma_{2}$ be the permutations associated to its transversal monodromies. It is well known that meridians around two smooth curves as $F_{1}$ and $F_{2}$ meeting 
transversaly (that is, loops bounding small transversal discs to $F_{i}$ ) commute. Since the monodromy is a group morphism, $\sigma_{1}$ and $\sigma_{2}$ must commute too.

Now, if we make an extra blowup at this point, a new divisor $F_{3}$ will appear, whose permutation corresponding to the transversal monodromy will be the composition of $\sigma_{1}$ and $\sigma_{2}$ (the order doesn't really matter, since they commute).

By successive blowing ups we can obtain divisors whose corresponding transversal permutation is $\sigma_{1}^{n} \cdot \sigma_{2}^{m}$ for every positive integers $n, m$. When $n$ and $m$ are multiples of the order of $\sigma_{1}$ and $\sigma_{2}$, we will obtain a divisor with trivial permutation.

So we have proven the following:

Theorem 5.2. Let $\pi: \mathbb{C}^{3} \rightarrow \mathbb{C}^{2}$ be a linear projection such that $\left.\pi\right|_{(X, \overline{0})}$ is finite. There always exist an arc $\gamma$ for which the curve $(X, \overline{0}) \cap \pi^{-1}(\gamma(\mathbb{D}))$ has as many irreducible components as the degree of the cover $\left.\pi\right|_{X}$.

Proof. Just take $\gamma$ to be the contraction of a smooth arc transverse to a divisor with trivial permutation.

Remark 5.3. A divisor with trivial permutation can always be obtained by blowing up a generic point in any component of the exceptional divisor and then keep blowing up the point at the normal crossing of the divisors that appears. In that case the sequence of permutations that appear are consecutive powers of the original one.

This implies that there are arcs with totally reducible arc-sections with arbitrary tangent cone.

Example 5.4. For the case $z^{d}=f(x, y)$, if $x=0$ is a generic line for $f(x, y)=0$ (i.e. not tangent to it), for the projection to the $X Y$-plane, the arc $\left(t^{d+1}, t^{d}\right)$ gives a reducible arc-section. In this case this is easy to check since $z^{d}-f\left(t^{d+1}, t^{d}\right)=0$ has tangent cone $z^{d}-t^{k d}=0$ with $k$ the multiplicity of $f(x, y)=0$, which factorizes in d factors.

\subsection{The generic arc-section}

Let $(X, \overline{0})$ be a surface germ in $\left(\mathbb{C}^{3}, \overline{0}\right)$. For a given projection $\pi: \mathbb{C}^{3} \rightarrow \mathbb{C}^{2}$ with $\left.\pi\right|_{X, \overline{0}}$ finite we will say that a generic arc is a smooth arc not tangent to the discriminant $\Delta$ of $\left.\pi\right|_{X, \overline{0}}$.

All generic arcs give the same associated monodromy. Moreover, this is the case for any projection in a direction of the open set $\Omega \subset \operatorname{Grass}_{1}\left(\mathbb{C}^{3}\right)$ of directions that are Zariski equisingularity. This is because we can deform one generic arc to the other. Then, one is transitive if and only if all of them are.

Given a singular arc transverse to the discriminant, then its monodromy is a power of the monodromy of the generic smooth arc by arguments in Section 5. Then, if a singular arc gives rise to an irreducible arc section, its monodromy must be transtive and since it is the power of the monodromy of the generic arc, the monodromy of the generic arc should be also transitive. That is:

Corollary 5.5. If an arc not tangent to $\Delta$ gives an irreducible arc-section, then all smooth arcs not tangent to the discriminant do.

Corollary 5.6. If the generic arc gives an irreducible arc section, then either the tangent cone $C_{X, \overline{0}}$ is a line (with certain multiplicity) or the tangent cone is a union of lines and we are projecting from the singular point of $C_{X, \overline{0}}$. 
Proof. Looking at the cases $(a)-(e)$ in Remark 4.7 we see that these two cases are the only ones that allow irreducible arc sections in any generic direction.

Corollary 5.7. If $C_{X, \overline{0}}$ is not a line or a union of lines and there is an irreducible arc-section over an arc $\gamma$, then the arc $\gamma$ is tangent to $\Delta$.

\section{Discriminant of the tangent cone coincides with the tangent cone of the discriminant}

After Corollary 5.7 we are interested in the tangent cone of $\Delta$ in order to know the tangent line of an arc with irreducible-arc section.

Let $(X, \overline{0})$ be a surface in $\left(\mathbb{C}^{3}, 0\right)$. We want to prove that when projecting in the direction of a point in a line $L$ as in the hypothesis of Lemma 4.1, we can know in advance the only possible tangent line for an arc with irreducible arc-section.

Following [1, we consider the Nash modification $\widetilde{X}$ that is the closure in $\mathbb{C}^{3} \times \operatorname{Grass}_{2}\left(\mathbb{C}^{3}\right) \approx \mathbb{C}^{3} \times \widetilde{C P}^{2}$ of $\left\{\left(x, T_{x} X\right): x \in X\right\}$. The projection of $\widetilde{X}$ onto the first factor is called the Nash blowup

$$
\mathcal{N}: \widetilde{X} \rightarrow X
$$

and the one onto the second, the gauss map

$$
\widetilde{\mathcal{G}}: \widetilde{X} \rightarrow \overleftarrow{C}^{2} .
$$

We recall that $\mathcal{N}^{-1}(0) \subset\{0\} \times \check{\mathbb{C P}}^{2}$ is the union of $\check{C}_{X, \overline{0}}$, that denotes the dual curve of the tangent cone $C_{X, \overline{0}} \subset \mathbb{C P}^{2}$, and some lines that correspond to pencils of planes passing through the exceptional tangent lines.

Every point in $\mathcal{N}^{-1}(0) \subset\{0\} \times \check{\mathbb{C P}}^{2}$ corresponds to a hyperplane in $\left(\mathbb{C}^{3}, 0\right)$, limit of tangent hyperplanes to $(X, \overline{0})$. In particular, a point in the dual of the tangent cone, represents the plane in $\mathbb{C}^{3}$ induced by the tangent line to its dual point in $C_{X, \overline{0}} \subset \mathbb{C P}^{2}$.

Proposition 6.1. Assume the projection direction is not an exceptional tangent. Then, the tangent of the discriminant $\Delta$ coincides with the discriminant of $\left.\bar{\pi}\right|_{C_{X, \overline{0}}}$.

Proof. The projection direction $p_{\pi}$ gives a line $T_{\pi}$ in $\check{C P P}^{2} \approx \mathbb{C P}^{2}$ that corresponds to the pencil of hyperplanes that contains the direction.

The set $\mathcal{N}\left(\mathcal{G}^{-1}\left(T_{\pi}\right)\right)$ gives the polar curve $\Gamma$ of the projection. Since $T_{\pi}$ is not the pencil of an exceptional tangent, we have that $\mathcal{N}\left(\mathcal{G}^{-1}\left(T_{\pi}\right)\right)$ has one dimensional component in $(X, \overline{0})$. This is because $T_{\pi}$ is not the pencil of an exceptional tangent line and because $\mathcal{G}^{-1}\left(T_{\pi}\right)$ is defined by 1 equation in a surface $\widetilde{X}$ and $\mathcal{G}^{-1}\left(T_{\pi}\right)$ is not contained in $\mathcal{N}^{-1}(0)$.

It is immediate that whenever we have a point in the discriminant of $\bar{\pi}$, that is, whenever there exists $q \in C_{X, \overline{0}}$ such that $\overline{q p}_{\pi}$ is a line tangent to $C_{X, \overline{0}}$ at $q$, we have an intersection point of $T_{\pi} \cap \check{C}_{X, \overline{0}}$, that is a limit tangent hyperplane to $(X, \overline{0})$ that contains $\left\langle p_{\pi}>\right.$. And reciprocally, an intersection point of $T_{\pi} \cap \check{C}_{X, \overline{0}}$ gives a line tangent to $C_{X, \overline{0}}$ passing by $p_{\pi}$. 
Finally, the tangent line at the origin of every branch of the discriminant $\pi(\Gamma)$ is not zero and is given by limits of tangent lines out of the origin. These lines are the image by $\bar{\pi}$ of the tangent lines of the polar curve, which are contained in the hyperplanes corresponding to points of $T_{\pi}$. Then, since the tangent line is not zero, it has to be in the discriminant or $\bar{\pi}$.

Corollary 6.2. Assume $C_{X, \overline{0}}$ is not a line or a union of lines. Assume moreover that the tangent lines to $C_{X, \overline{0}}$ at the points corresponding to exceptional tangents of $X$ meet $C_{X, \overline{0}}$ in at least two points.

In this case, for any finite linear projection $\pi$, the tangent line of an arc in $\mathbb{C}^{2}$ with irreducible arc-section corresponds to a point in the discriminant of $\left.\bar{\pi}\right|_{C_{X, \overline{0}}}$, with $\bar{\pi}: \mathbb{P}^{2} \rightarrow \mathbb{P}^{1}$.

Proof. If there is an irreducible arc section, by Lemma 4.1 there should exists a line $L$ in $\mathbb{C P}^{2}$ that intersects $C_{X, \overline{0}}$ in only one point. Since $C_{X, \overline{0}}$ is not a line or union of lines, $L$ should be a tangent line to $C_{X, \overline{0}}$ at certain point $p$ that does not meet other points. Moreover, by Corollary 5.7 the arc has to be tangent to the discriminant.

By hypothesis $p$ does not correspond to an exceptional tangent to $X$, so, by Proposition 6.1, the tangent to the discriminant coincides with the discriminant of $\left.\bar{\pi}\right|_{C_{X, \overline{0}}}$. Then we can conclude.

Examples where the hypothesis of Corollary 6.2 are satisfied are any surface with $C_{X, \overline{0}}$ smooth, since it is known that there is no exceptional tangents in this case (see (2.2.1) in [1]).

\section{Algorithm to find an irreducible arc-section.}

Let $(X, \overline{0})$ be a surface germ in $\left(\mathbb{C}^{3}, \overline{0}\right)$. Based on the previous sections, we get a general method to determine if a given surface germ projection $\pi:(X, \overline{0}) \rightarrow$ $\left(\mathbb{C}^{2}, 0\right)$ admits irreducible arc sections or not.

First of all, we find the discriminant $\Delta$. The following steps are:

1. Compute an embedded resolution of singularities of $\Delta, \phi:\left(\widetilde{\mathbb{C}}^{2}, E\right) \rightarrow$ $\left(\mathbb{C}^{2}, \overline{0}\right)$. Applying the same sequence of blowups, we get a map $\widetilde{\phi}$ : $(\widetilde{X}, \bar{E}) \rightarrow(X, \overline{0})$.

2. For each normal crossing along the total transform of $\Delta$, that is, for every intersection of two branches $B_{1}, B_{2}$ in $\phi^{-1}(\Delta)$, get two small smooth disks $D_{1}, D_{2}$ such that:

- $D_{i}$ is centered in a point of $B_{i}$, is transversal to it, and touches no other component of $\phi^{-1}(\Delta)$

- There exists a point $q$ in the intersection of the boundaries $\partial D_{1} \cap \partial D_{2}$

- Both disks are inside a small polydisc centered in the normal crossing point, that meets no other component of $\phi^{-1}(\Delta)$.

3. Consider $\pi \circ \widetilde{\phi}$ restricted to the preimage of $\partial D_{1}$ and $\partial D_{2}$ as in 2 and compute the permutations $P_{1}$ and $P_{2}$ of the corresponding monodromy based in $q$. 
4. The projection $\pi$ allows an irreducible arc section if and only if for some $P_{1}, P_{2}$ as before, the group generated by them contains a transitive element.

Example 7.1. Consider the surface germ given by $z^{4}-4 x z+3 y^{2}=0$, projected along the $z$ direction. Note that it is an isolated singularity, and hence it must be irreducible. Its discriminant is given by the equation $\left(y^{3}-x^{2}\right)\left(y^{3}+x^{2}\right)=0$.

We compute a resolution of singularities by the sequence of blowups in figure 1

We get three exceptional divisors and two branches. Let's focus for instance in the normal crossing at the origin of the last chart. The two discs $B_{1}, B_{2}$ can be taken as $x=\frac{1}{2},\|y\| \leq \frac{1}{2}$ and $\|x\| \leq \frac{1}{2}, y=\frac{1}{2}$ respectively, so the base point $q$ would be $\left(\frac{1}{2}, \frac{1}{2}\right)$. The composition of the blow-up transformations applied to the equation of $X$ is $z^{4}-4\left(x^{2} y^{3}\right) z+3\left(x y^{2}\right)^{2}=0$.

To compute the permutation around the boundary of the disk, we parametrize the disk as $\left(\frac{1}{2}, \frac{t}{2}\right)$ and substitute, obtaining $z^{4}-\frac{1}{8} t^{3} z+\frac{3}{64} t^{4}$. This is a squarefree homogenous equation in two variables, so it factors as the product of four linear forms. That is, the arc section corresponding to this arc is a union of four lines, and the corresponding monodromy has a trivial permutation.

The other disk can be parametrized as $\left(\frac{t}{2}, \frac{1}{2}\right)$ and after composing, we get the equation $z^{4}-\frac{1}{8} t^{2} z+\frac{3}{64} t^{2}=0$. The braid monodromy of this curve around the boundary of the unit disk is given by the braid shown in Fig 2

This braid can be expressed in the Artin presentation (see for instance [2]) as $\left(\sigma_{0} \sigma_{2} \sigma_{1}\right)^{2}$. Its permutation is $(1,4)(2,3)$. In this case, the group generated by these two permutations only has two elements, none of which is transitive.

We can repeat these computations with the rest of the crossings. In this case, one of the permutations will always be trivial (since they correspond to the first divisor we have computed). Similar computations show that the rest of the permutations we obtain are $(3,4),(3,4)$ and $(1,4,2)$ respectively. None of them are transitive and the group generated by them and the trivial permutation will not contain any transitive element. That is: in this case, there is no irreducible arc section.

In fact, not every possible branch must be checked. It is enough to study the branches $\Delta_{i}$ of $\Delta$ whose tangent line corresponds to a point in $\mathbb{C P}^{2}$ which generates, joined with the projection point, a line $L$ that meets $C_{X, \overline{0}}$ only in one point (see Lemma 4.1). Moreover, we have a necessary condition:

Lemma 7.2. Consider an arc that can be deformed to a branch $\Delta_{i}$ of the discriminant without intersecting the discriminant outside $\Delta_{i}$, and whose corresponding arc-section is irreducible, then a parametrizaton of $\Delta_{i}$ also has an irreducible arc-section.

Proof. Let $\gamma_{i}:(\mathbb{D}, 0) \rightarrow\left(\mathbb{C}^{2}, 0\right)$ be such an arc, and $\delta:(\mathbb{D}, 0) \rightarrow\left(\mathbb{C}^{2}, 0\right)$ be a parametrization of $\Delta_{i}$. Take a continuous deformation $T:(\mathbb{D}, 0) \times I \rightarrow\left(\mathbb{C}^{2}, 0\right)$ such that $\left.T\right|_{(\mathbb{D}, 0) \times\{0\}}=\gamma_{i},\left.T\right|_{(\mathbb{D}, 1) \times\{0\}}=\delta_{i}$, and $T(\partial \mathbb{D} \times[0,1)) \cap \Delta=\emptyset$.

The restriction of this deformation to the boundary of $\mathbb{D}$ gives a continuous deformation from the closure of the braid corresponding to $\gamma_{i}$ to the closure of the braid corresponding to $\Delta_{i}$. Since the arc section of $\gamma_{i}$ is irreducible, its braid is transitive, or equivalently, its closure is a knot. Since the deformation of the arcs does not intersect the discriminant, the different steps of the deformation 


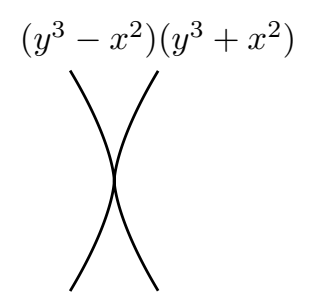

$$
\uparrow(x, y) \mapsto(x y, x)
$$

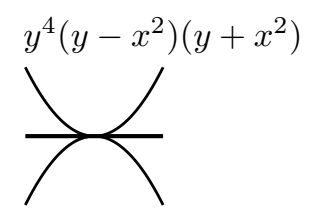

$$
\uparrow(x, y) \mapsto(x, x y)
$$
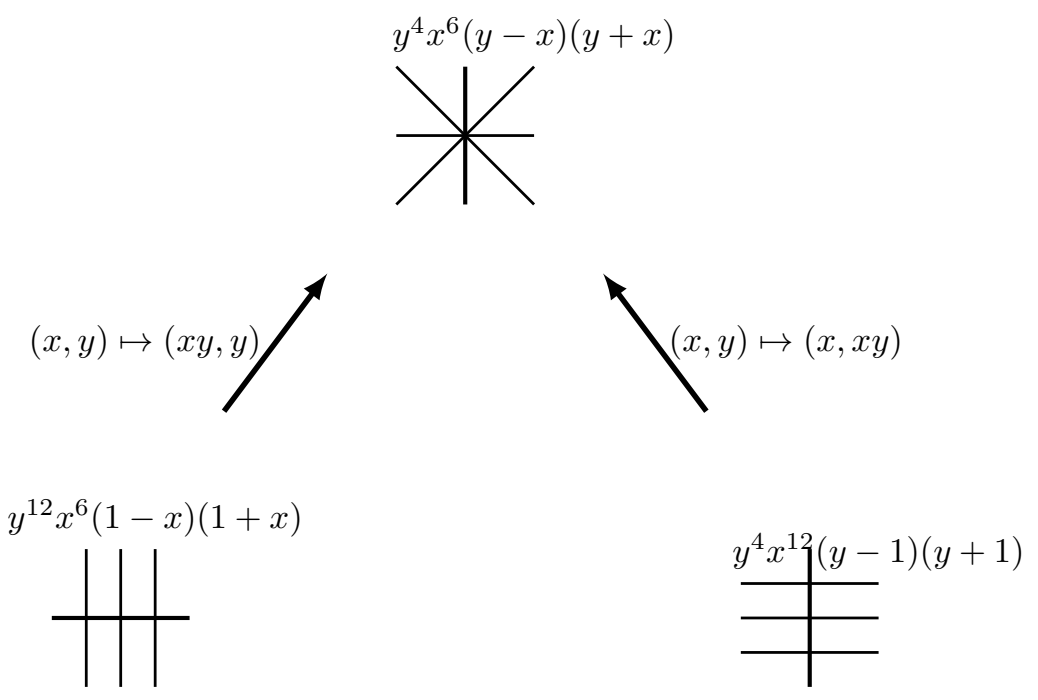

Figure 1: Sequence of blowups to resolve the singularities of the discriminant. 


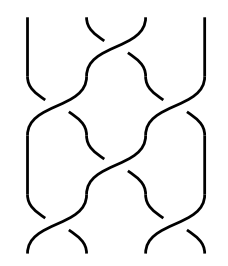

Figure 2: The braid $\left(\sigma_{0} \sigma_{2} \sigma_{1}\right)^{2}$

(except for the last one) are knots isotopic to the original one. So, the closure of the braid corresponding to $\Delta_{i}$ is the image of a knot by a continuous function, and hence, it must have also only one connected component. Hence the braid corresponding to $\Delta_{i}$ must also be transitive.

This phenomenon happens, for instance, with the arcs that are parallel to the branch $\Delta_{i}$ in the chart centered at the normal crossing between $\Delta_{i}$ and the rest of the exceptional divisor. So if we are interested in finding irreducible arc sections, in the previous method we can skip the cases around branches of the discriminant whose parametrization doesn't give an irreducible arc section.

\subsection{Sections over arcs contained in the discriminant}

We finish with an example where the behaviour over a parametrization of a branch of the discriminant changes under a small change of the projection.

Example 7.3. Consider the surface germ given by $z^{3}-(x-y)(x+y)(x-$ $2 y)(x+2 y)$. If we project in the $z$ direction, the discriminant is formed by the four lines $x=y, x=-y, x=2 y$ and $x=-2 y$. Over a generic arc such as $x=0$ we get an irreducible arc-section (with equation $z^{3}-y^{4}$ ).

Over each point of the discriminant, there is only one point of the surface. So, if we take an arc that parametrizes one component of the discriminant, the arc section that we get is a multiple line.

Now consider a line that doesn't go through the origin, say $x=\epsilon$ for some small $\epsilon>0$. It meets transversally the discriminant in four points (one for each component) $(\epsilon, \epsilon),(\epsilon,-\epsilon),(\epsilon, 2 \epsilon),(\epsilon,-2 \epsilon)$. Over each of these four points, there is only one preimage. It is easy to check that the local equation for the arc section over the arc that parametrizes the line $x=\epsilon$ centered at any of these four points is a vertical flex.

Now we make a small deformation of the projection (e.g. make the change of variables $y=y+\delta z$ for some small $\delta>0)$. Then the four lines of the discriminant get deformed into 4 cusps. Let's see the arc section for an arc that parametrizes one of them. To do so, consider again the section over the line $x=\epsilon$. It is the same small change of variables aplied to the previous curve with four vertical flexes. If we rotate slightly a vertical flex, it splits in two different simple vertical tangencies. That means that over each point of the discriminant, there will be two points of the surface: one with multiplicity 2 and other with multiplicity 1. Hence, the arc section corresponding to a parametrization of one of the cusps will contain a component with multiplicity 2 and another with multiplicity 1 . That is, it will be reducible. 

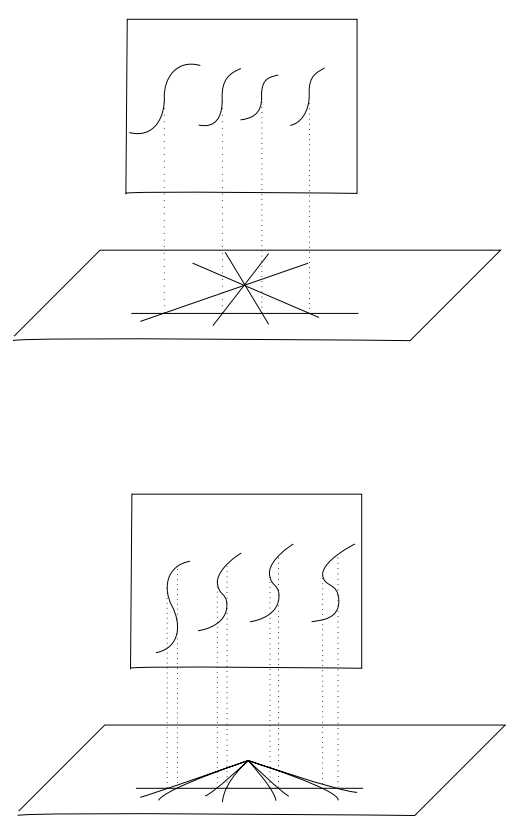

Figure 3: A rotation causes the discriminant to unfold from lines to cusps.

Note that both projections are non-transversal to the surface and have different discriminants but the generic arc section is irreducible in both cases.

If we apply the previous algorithm to this example, we can find a smooth arc whose arc section has 3 components, just by making it have contact 3 with one of the lines of the discriminant. An explicit equation could be $\left(t-t^{3}, t+t^{3}\right)$; then the equation for the arc section is $-12 t^{10}-40 t^{8}-12 t^{6}+z^{3}$, which is analytically equivalent to $-12 t^{6}+z^{3}$. Clearly this factorizes as the product of three components.

\section{References}

[1] Lê D ung Tráng and Bernard Teissier. Sur la géométrie des surfaces complexes. I. Tangentes exceptionnelles. Amer. J. Math., 101(2):420-452, 1979.

[2] E. Artin. Theory of braids. Ann. of Math. (2), 48:101-126, 1947. 